\title{
Solvent Reorganization Energies Measured by an Electron Transfer Reaction in Supercritical Ethane
}

\author{
Kenii Takahashi ${ }^{a^{*}}$, Wu Fenga, Sadashi Sawamura a and Charles D. Jonah ${ }^{*}$ \\ [a] Faculty of Engineering, Hokkaido University, Sapporo 060, Japan \\ [b] Chemistry division, Argonne National Laboratory, 9700 S. Cass Ave., Argonne, IL 60439, USA \\ kenji@hune.hokudai.ac.jp (+81-11-706-6675), Jonah@anlchm.chm.anl.gov(+1-630-252-4993)
}

The intermolecular electron transfer reaction between a biphenyl anion and pyrene in supercritical ethane was studied using pulse radiolysis. Second-order electron transfer rates were found to be of the order of $10^{11} \mathrm{M}^{-1} \mathrm{~s}^{-1}$. The rate constants appear to be approximately constant over the pressure range 55-133 bar. Two possibilities are discussed that could explain the present results: solvent clustering; or a dependence of the solvent reorganization energy on pressure. Reorganization energies $E_{r}$ of non-polar supercritical ethane were estimated from the observed rate constant using the modified Marcus equation. $E_{r}$ may be larger than normally expected for nonpolar solvents because of density fluctuations.

\section{Introduction}

It is well known that density fluctuations [1] and clustering of solvent molecules [2-8] are observed in supercritical fluids(SCFs). The local density of SCFs around a solute may be significantly greater than the bulk density of fluid not only in polar fluids [9] but also in non-polar fluids [10]. The number of molecules around a solute is significantly changed near the critical pressure [11]. Recently, Matyushov and Schmid[12] have theoretically studied solvent reorganization energy $E_{r}$ arising from charge separation and recombination reactions in non-polar fluids. According to their results, the $E_{r}$ in non-polar solvent is not zero but can be represented as the sum of two terms that arise from liquid polarization and density reorganization, with the latter component being of much greater importance.

For this reason we felt that electron transfer (ET) reactions in non-polar SCFs might show a new aspect of the solvent reorganization energy in non-polar fluids. While radical reactions have been studied in SCFs[13-16 for example], we are unaware of any ET reactions that have been studied in SCFs.

In this work we present the intermolecular ET reactions in non-polar SCFs. We chose to use ethane as a non-polar solvent because it was relatively unreactive to the ions formed and a supercritical fluid could be formed conveniently. Biphenyl anion and pyrene were used as an electron donor and acceptor, respectively. The solvent reorganization energies were estimated using the measured ET rates.

\section{Materials and Methods}

High pressure equipment Experiments were run with a cylindrical stainless-steel high pressure cell (Takagi equipment CO., LTD., $4 \mathrm{~cm}$ in O.D., $9.5 \mathrm{~cm}$ in length). The $1 \mathrm{~cm}$ thick suprasil windows were mounted to the cell using Teflon o-rings. The optical path length is 6 $\mathrm{cm}$ and the cell capacity is $4.2 \mathrm{~cm}^{3}$. The temperature of the cell was maintained constant to $\pm 0.1^{\circ} \mathrm{C}$ at $35^{\circ} \mathrm{C}$ $\left( \pm 0.5^{\circ} \mathrm{C}\right)$ using an Omega temperature controller
(Model CN1001RTD), a cartridge heater and a platinum resistance thermometer. The thermometer was installed into the cell so that it is in contact with the fluid. Pressures were generated using a JASCO HPLC pump (Model PU-980, with a flow controller and pressure sensor). The fluid pressure was monitored with Cole-Parmer digital pressure meter (Model 7350-38) and pressure transmitter (Model K1, $3000 \mathrm{psi}$ ) .

Sample preparation Small amounts of stock solutions of biphenyl (BPh) (Aldrich 99\%) and pyrene (Py) (Aldrich $99 \%$ ) in ethanol, respectively $0.2 \mathrm{M}$ and 0.01 $M$, are put into the optical cell using microsyringes and the solvent was removed by heating the cell. After the evaporation of the ethanol, the cell was connected to the HPLC pump through a series of valves. To minimize the presence of oxygen, the entire apparatus was purged with ethane at low pressure. After the pressure was set, the sample was mixed by a magnetic stirring bar. In a given experimental run, the molarity was held constant while the pressure was varied. At higher concentrations, experiments could not be run at lower pressures because of the limited solubility.

The density of supercritical (SC) ethane was calculated with the Peng-Robinson cubic equation of state[17]. The saturated solubility of BPh and Py in $S C$ ethane were obtained by measurement of the UV absorption spectrum and assuming the extinction coefficients of BPh and Py are the same as those in ethanol. No shift of the absorption spectrum was detected over the pressure range studied.

Pulse radiolysis We used the pulse radiolysis method to study ET reactions in SC ethane. A conventional pulse radiolysis system was used[18]. Electron pulses from the Argonne Electron Linear Accelerator, about $30 \mathrm{ps}$ in duration, $20 \mathrm{MeV}$ energy, irradiated the solution. Both a short flash lamp and pulsed $75 \mathrm{~W}$ xenon lamp were used as light sources for determining the transient absorption, depending on the time scale. The light was detected using photomultiplier (Hamamatsu 


\section{DISCLAIMER}

This report was prepared as an account of work sponsored by an agency of the United States Government. Neither the United States Government nor any agency thereof, nor any of their employees, make any warranty, express or implied, or assumes any legal liability or responsibility for the accuracy, completeness, or usefulness of any information, apparatus, product, or process disclosed, or represents that its use would not infringe privately owned rights. Reference herein to any specific commercial product, process, or service by trade name, trademark, manufacturer, or otherwise does not necessarily constitute or imply its endorsement, recommendation, or favoring by the United States Government or any agency thereof. The views and opinions of authors expressed herein do not necessarily state or reflect those of the United States Government or any agency thereof. 


\section{DISCLAIMER}

Portions of this document may be illegible in electronic image products. Images are produced from the best available original document. 
R928) and monochromator system or photodiode (CD 10) and bandpass filter system. The signal from the detector was digitized using a Tektronix SCD 5000.

\section{Results and Discussion}

Fig. 1 shows typical transient spectra obtained for the solution of $\mathrm{BPh}$ and $\mathrm{Py}$ in SC ethane. The concentration of $\mathrm{BPh}$ is considerably larger than that of Py so that most of the electrons formed by the ionizing radiation from the solvent will react with the $\mathrm{BPh}$. The peak near $640 \mathrm{~nm}$ is attributed to absorption of biphenyl anion (BPh-) and its magnitude decreases with time. The absorption around $480 \mathrm{~nm}$, which builds up at the same rate as the $640 \mathrm{~nm}$ absorption decays, is assigned to pyrene anion $\left(\mathrm{Py}^{-}\right)$, which is formed by the intermolecular electron transfer reaction.

Fig. 2 shows transient kinetics at 640 and $480 \mathrm{~nm}$, respectively, corresponding to $\mathrm{BPh}^{-}$and $\mathrm{Py}$. Under the present experimental conditions the concentration of $\mathrm{BPh}^{-}$produced can be estimated as ca $10^{-6} \mathrm{M}$ by assuming the extinction coefficient of $\mathrm{BPh}$ - in SC ethane is the same as that in ethanol. The concentration of Py was adjusted to $100,200,300,400$ and $500 \mu \mathrm{M}$ and the ET reaction was studied under pseudo-firstorder reaction conditions. The pseudo-first-order reaction rates were calculated by simultaneously fitting the decay of $\mathrm{BPh}^{-}$and growth of $\mathrm{Py}^{-}$. The second order rate constants were extracted from the pseudofirst-order rates, and are displayed as a function of the pressure of SC ethane in Fig.3a.

If there are no electrostatic interactions between the reactants, the rate of an ET reaction can be described by equation[19].

$$
k_{o b s}=\frac{k_{d}}{1+.2 \exp \left(E_{r}\left(1+\frac{\Delta G}{E_{r}}\right)^{2} / 4 R T\right)}
$$

where $k_{d}$ is a diffusion controlled rate constant, $-\Delta G$ free energy change $(0.52 \mathrm{eV}$ for the present system[18]) and $E_{r}$ reorganization energy. The diffusion-controlled rate for a reaction and distance $r$ can be described by Eqn. 2 and is plotted in Fig.3a.

$k_{d}=4 \pi r D N_{a}$

where $N_{a}$ is Avogadro's number, $r$ is the reaction distance and assumed as a sum of the donor and the acceptor radii, $D$ the sum of the diffusion coefficients of the reactants. Values of $D$ were calculated by hydrodynamic theory in which the hydrodynamic radius of $\mathrm{BPh}^{-}$and $\mathrm{Py}$ are assumed to be the same as naphthalene as a model aromatic. The viscosity of SC ethane was calculated using an equation proposed by Younglove[20]. The calculated $k_{d}$ may be an underestimate because the reaction radius may be larger than the sum of the donor and acceptor radii; however the relative dependence of $k_{d}$ on pressure is independent

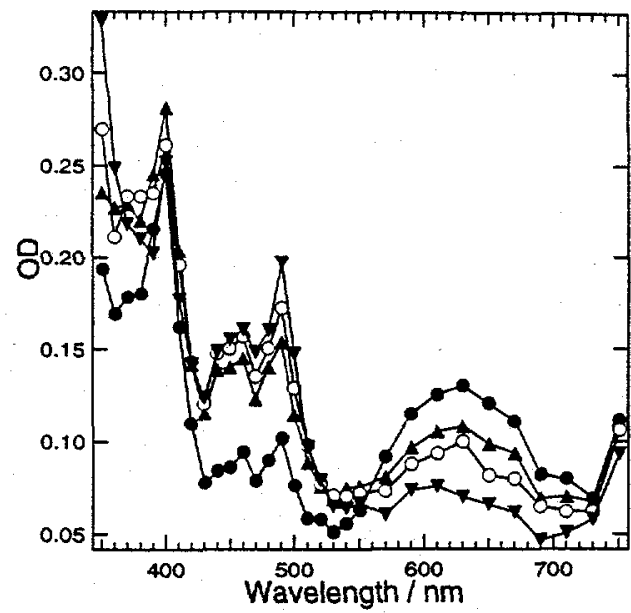

Figure 1. Transient absorption spectra monitored at 3 $(\bullet) 6(\Delta) 10(0)$ and $15(\nabla)$ ns after the pulse. The concentrations of $\mathrm{BPh}$ and $\mathrm{Py}$ are 9 and $0.7 \mathrm{mM}$, respectively. The pressure was 130 bar and temperature $35^{\circ} \mathrm{C}$.

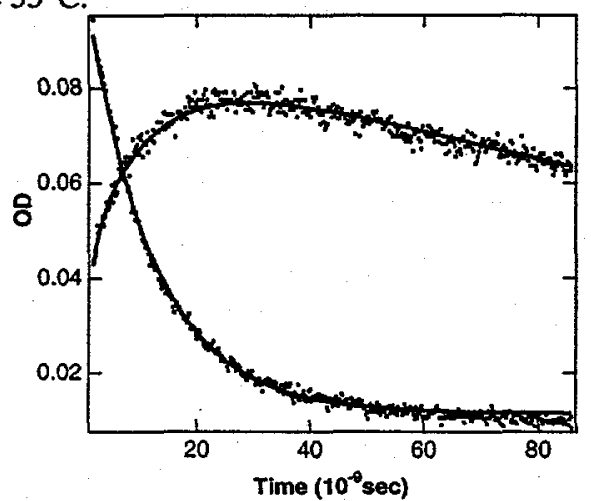

Figure 2. Transient kinetics at 640 and $480 \mathrm{~nm}$. The pressure is 77 bar and temperature was $35^{\circ} \mathrm{C}$. The concentrations of Py and BPh are $400 \mu \mathrm{M}$ and 7 $\mathrm{mM}$, respectively.

of the reaction radii.

On examination of Fig. 3a, one observes that the rate constants appear to be approximately constant over the pressure range 50-140 bar. This occurs despite the fact that the diffusion-controlled rate changes markedly over the pressure range (see the solid curve in Fig. 3a). Two possible explanations for this result are: 1) the viscosity of the SCFs does not determine the diffusion rate and thus diffusion-controlled rate is not what is expected or 2) the solvent reorganization energy $E_{r}$ changes with pressure. We will discuss these two possibilities separately.

In a recent publication[21], it has been shown that the rotational diffusion time of a fluorescent species is considerably slower than one would predict from the calculated viscosity of the fluid at lower pressures. Also the difference between predicted and measured 
Table 1

Solvent reorganization energy in nonpolar liquids

\begin{tabular}{lll}
\hline & solvent & $E_{r}(\mathrm{eV})$ \\
\hline Closs et al.[18] & isooctane & 0.15 \\
Gould et al.[23] & $\mathrm{n}-\mathrm{C} 6 \mathrm{H} 12$ & 0.14 \\
& $\mathrm{CCl} 4$ & 0.16 \\
Cortes et al.[24] & $\mathrm{n}-\mathrm{C} 6 \mathrm{H} 12$ & $0.33-0.51$ \\
& $\mathrm{CCl} 4$ & $0.31-0.46$
\end{tabular}

reorientation times decreased as the pressures increased. The authors suggested that this result could be due to either a 1) cluster of solvent molecules around the excited state that inhibits the rotation of the molecule or 2) a clustering of solvent molecules with the solute molecule where this cluster moves as a group (and thus more slowly). Certainly the second suggestion would explain the present data.

Simulations by Ganapathy, et al. have shown that reaction rates will not follow the Smoluchowski rate expression in supercritical fluids if the reaction probability is small.[22] A large solvent reorganization energy could lead to similar apparent results as a low reaction probability.

Morita and Kajimoto [10] have reported a clustering in nonpolar supercritical fluids. Their results show that the solvatochromic shift is much larger than expected from Onsager's theory. The bathochromic shifts can be attributed to the solvent aggregation around a solute even in nonpolar ethane. These results are consistent with the present data.

The second alternative, a change in the solvent reorganization energy $E_{r}$ would also explain the data. Eqn. 1 shows that a shift in $E_{r}$ alters the rate constant. Fig. 3b shows the two calculated values of $E_{r}$ as a function of pressure using the assumed values for the diffusion-controlled rate. Because of the quadratic dependence of the rate on $E_{r}$ there are two values for $E_{r}$ that would explain a particular experimental rate constant. These results can be compared with the literature results for nonpolar liquids, which are summarized in Table 1. The previous results are smaller than either curve given in Fig $3 \mathrm{~b}$ except the results by Cortes et al.[24] (The interpretation of those results is uncertain, in that the authors refer to these values as unphysically large.) There are several possible explanations for the relatively large value of $E_{r}$ determined from the present experiment. As Miller has shown, there can be a low energy twisting motion of BPh that would contribute approximately $0.15 \mathrm{eV}$ [25]. If this value is added to the literature values for $E_{r}$ the lower curve is only slightly higher than suggested by previous work. In addition, experimental studies[1] have shown the existence of large fluctuations near the critical point in supercritical fluids. Theoretical [12] studies have suggested that such fluctuations could increase
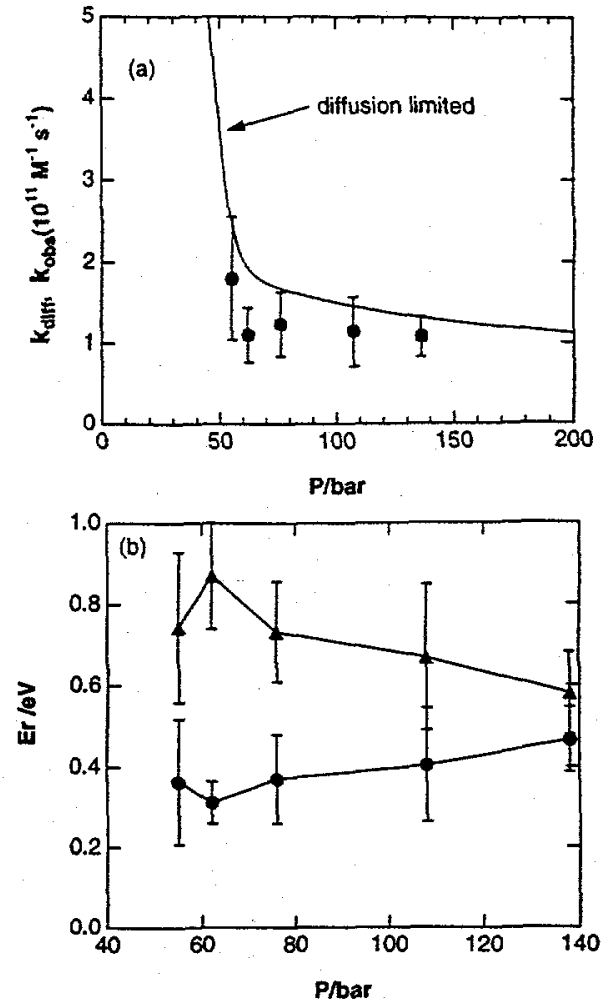

Figure 3. (a) Points are the ET rate constants in SC ethane as a function of total pressure at $35^{\circ} \mathrm{C}$. The solid line is the calculated diffusion-controlled rate constant $k_{d}$ as a function of pressure (see text).

(b) Calculated solvent reorganization energy based on Eqn. 1. See text for details.

the value of $E_{r}$

As stated above, the reaction radius that we used to calculate $E_{r}$ is a lower limit. If the reaction radius were larger, the two $E_{r}$ curves would separate further. It seems unlikely that $E_{r}$ could be much lower than that calculated in the lower curve (considering the possibility of some energy due to a twist in the biphenyl anion); thus we expect that the estimated radius is reasonable. If however the mobilities are lower than assumed (possibly due to clustering), the lower curve for $E_{r}$ would move up while the upper curve would move down. This would be consistent with the possible increase in $E_{r}$ that could arise from the fluctuations in the supercritical ethane.

According to the work by Matyshov and Schmid[12], $E_{r}$ can be represented as the sum of two terms, $E_{p}$ and $E_{d} . E_{p}$ determines the solvent reorganization due to the polarization of the fluid. In contrast, $E_{d}$ represents the contribution from repacking the solvent near the core of the electron donor-acceptor complex. Using their results, we have calculated $E_{r}$ for ethane. In the calculation the cavity radius, the molecular polarizability and the solvent diameter were set as $5 \AA, 4.07$ $\AA^{3}$ and $3.7 \AA$, respectively. The value for $E_{r}$ is calculated to be $0.47 \mathrm{eV}$, which is very close to the values 
of $E_{r}$ at high pressure that is shown in Fig.3b.

In their theory [12], $E_{d}$ is much larger than $E_{p}$. If one replaces the solute-solvent distribution function by a step function, the value of $E_{d}$ is greatly reduced and $E_{r}$ is approximately the same as $E p$. Our molecular dynamics simulations[26] show that at low densities, the pair correlation functions are less like a step function than they are at higher densities. Thus $E_{d}$ will be much larger relative to $E_{p}$ at lower densities. This means that $E_{r}$ will be larger at lower densities than one might expect. To explore the dependence of $E_{r}$ on pressure will require further experiments utilizing reactions with different $\Delta G$ 's. It is clear that the pressure dependence of $E_{r}$ in SCFs will be an important topic of future research. New simulations that make use of molecular dynamics and quantum calculations and the use of polarizable solutes and solvents will provide important insights into the mechanisms.

\section{Conclusion}

We have measured electron transfer reactions between an anion and a neutral species in a nonpolar supercritical fluid. To our knowledge this is the first measurement of electron transfer reactions in supercritical fluids. These results have provided evidence for new phenomena that occur in these systems. The reactions do not show the pressure dependence that would be expected for simple electron transfer reactions where the diffusivity is defined by the characteristics of the bulk solvent. These effects could be ascribed to the dependence of the solvent reorganization energy $E_{r}$ on pressure. $E_{r}$ may be larger than normally expected for non-polar solvents because of density fluctuations. The results could also suggest that the diffusion coefficient is lower than would be expected in the supercritical fluids near the critical point.

Electron transfer reactions in supercritical fluids can provide both interesting tests of electron transfer models and the electron transfer reaction can provide new probes to processes in supercritical fluids. Future studies using a variety of electron transfer reactants with different $\Delta G$ 's will allow an exploration of $E_{r}$ in supercritical fluids.

\section{Acknowledgments}

The authors thank Mr. Don Ficht for operation and maintenance of the LINAC used in this work. A part of this work was supported by a grant-in-aid (No.06750782) from the Ministry of Education, Japan. Support of C. D. Jonah and experiments performed at Argonne were under the auspices of the Office of Basic Energy Sciences, Division of Chemical Science, US-DOE under contract number W-31-109-ENG-38. We would also like to thank John Miller for the use of the equipment and discussions.
References

1. Nishikawa, K.; Tanaka, I.; Amemiya, Y. J. Phys, Chem. 1996, 100, 418-421.

2. Brennecke, J. F.; Tomasko, D. L. ; Eckert, C. A. J. Phys. Chem. 1990, 94, 7692-7700

3. Kajimoto, O.; Futakami, M.; Kobayashi, T.; Yamasaki, K. J. Phys. Chem. 1988, 92, 1374-1352

4. Randolph, T. W.; Carlier, C. J. Phys. Chem. 1992, $96,5146-5151$

5. Roberts, C. B.; Chateauneuf, J. E.; Brennecke, J. F. J. Am. Chem. Soc. 1992, 114, 8455-8463

6. Smith,R. D.; Frye, S. L.; Yonker, C. R.; Gale,R. W. J. Phys. Chem. 1987, 91, 3059-3062

7. Sun, Y. P.; Fox, M. A. J. Phys. Chem. 1993, 97, 282-283

8. Yonker, C. R.; Smith, R. D. J. Phys. Chem. 1988, 92, 2374-2387

9. Kim, S.; Johnston, K. P. Ind. Eng. Chem. Res. 1987, $26,1206-1213$

10. Morita, A.; Kajimoto, O. J. Phys. Chem. 1990 , $94,6420-6425$

11. Eckert, C. A.; Ziger, D. H.; Johnston, K. P.; Kim, S. J. Phys. Chem. 1986, 90, 2738-2746

12. Matyushov, D. V.; Schmid, R. Molec. Phys. 1995, 84, 533-552

13. Roberts, C. B.; Zhang, J.; Chateauneuf, J. E.; Brennecke, J. F. J. Am. Chem. Soc. 1995, 117, 65536560

14. Ganapathy, S.; Randolph, T. W; Carlier, C.; O'Brien, J. A. Int J. Thermophys 1996, 17, 471-481

15. O'Brien, J. A.; Randolph, T. W.; Carlier, C.; Ganapathy, S. A. I. Ch. E. J. 1993, 39, 1061-1071

16. Zhang, J.; Connery, K. A.;Brennecke, J. F.; Chateauneuf, J. E. J. Phys. Chem. 1996, 100, 1239412402

17. Peng, D. Y.; Robinson, D. B. Ind. Eng. Chem., Fundam. 1976, 15, 59-64

18. Closs, G. L.; Calcaterra, L. T.; Green, N. J.; Penfield, K. W.; Miller, J. R. J. Phys. Chem. 1986, 90, 3673-3683

19. Eberson, L. Adv. Phys. Org. Chem. 1982, 18, 79185

20. Younglove, B. A.; Ely, J. F. J. Phys. Chem. Ref. Data 1987, 16, 577-798

21. Heitz, M. P.; Bright, F. V. J. Phys. Chem. 1996, $100,6889-6897$

22. Ganapathy, S.; O'Brien, J. A.; Randolph, T. W. J. Supercrit. Flu. 1996, 9, 51-55

23. Gould, I. R.; Noukakis, D.; Goodman, J. L.; Young, R. H.; Farid, S. J. Amer. Chem. Soc. 1993, $115,830-3831$

24. Cortes, J.; Heitele, H.; Jortner, J. J. Phys. Chem. $1994,98,2527-2536$

25. Miller, J. R.; Paulson, B. P.; Bal, R.; Closs, G. L. J. Phys. Chem. 1995, 99, 6923-6925

26. Takahashi, K.; Abe, K.; Sawamura, S.; Jonah, C.

D. submited to Chem. Phys. Lett. 